\section{A influência da atividade física nos quadros depressivos de pessoas idosas: uma revisão sistemática}

\section{The influence of exercise on depressive disorders of the elderly: a systematic review}

\section{Joanna Miguez Nery Gumarães ${ }^{1}$ \\ Célia Pereira Caldas ${ }^{2}$}

'Programa de Pós-Graduação em Saúde Coletiva, Departamento de Epidemiologia, Instituto de Medicina Social, Universidade do Estado do Rio de Janeiro (UERJ)

2Departamento de Saúde Pública (Faculdade de Enfermagem/UERJ), Programa de Pós-Graduação em Ciências Médicas (FCM/UERJ); Universidade Aberta da Terceira Idade (UnATI/UERJ)

\section{Resumo}

Introdução: Transtornos depressivos têm alta prevalência entre idosos e estão associados a um impacto negativo em seu estado de saúde e qualidade de vida. A associação entre a prática de atividades físicas e a depressão vem sendo investigada pela literatura, mas seus resultados têm se mostrado inconclusivos. Objetivos: Avaliar estudos que analisaram o papel da atividade física nos diferentes níveis de prevenção dos transtornos depressivos (depressão maior, depressão menor e distimia) em idosos. Material e Métodos: Foi realizada uma revisão sistemática de literatura na base de dados MEDLINE e na biblioteca virtual SciELO, referente aos anos de 1995 a 2004. Foram analisados aspectos como o tipo de intervenção ou observação, tempo de seguimento, instrumento utilizado para medir a depressão, tamanho amostral, perdas de seguimento e ajustamento das variáveis de confundimento. Resultados: Quinze artigos foram selecionados, sendo oito estudos controlados-randomizados, cinco estudos transversais e dois estudos de coorte. Os aspectos metodológicos analisados variaram entre os trabalhos avaliados. Muitos estudos sugerem uma relação de associação entre a prática de atividades físicas e a depressão em idosos. No entanto, o tipo e a força de associação variaram nas diferentes amostras. Conclusão: A literatura científica parece, de maneira geral, sustentar os efeitos benéficos da atividade física e do exercício na depressão em pessoas idosas. Apesar disso, são necessárias mais pesquisas sobre o assunto e que apresentem um maior rigor metodológico.

Palavras-chave: Revisão sistemática. Epidemiologia. Idosos. Depressão. Atividade física.

Correspondência: Joanna M. N. Guimarães. Rua Sacopã, 191/102 - Lagoa - Rio de Janeiro, Brasil CEP 22471-180. E-mail: joannaguimaraes@hotmail.com 
Abstract

Introduction: Depressive disorders have a high prevalence among the elderly and are associated with a negative impact on their health status and quality of life. There are investigations in the literature on the relationship between exercising and prevention of depression, but results have been inconclusive. Objectives: To evaluate studies that analyzed the role of exercise on the prevention of different levels of depressive disorders (major depression, minor depression and dysthymia) in the elderly. Methods: A systematic review of the literature was conducted in the MEDLINE database and in the SciELO virtual library, for the years of 1995 to 2004. Parameters such as the type of intervention or observation, length of follow-up, instrument utilized for measuring depression, sample size, losses during follow-up, and adjustments for confounding variables were analyzed. Results: Fifteen articles were selected: eight were controlled randomized studies, five were cross-sectional studies and two were cohort studies. The methodological parameters analyzed differed among the studies evaluated. Many studies suggested an association between exercise and the prevention of depression in the elderly. However, the type and strength of the association differed among the different samples. Conclusion: The scientific literature in general, seems to support the beneficial effects of physical activity and exercise in preventing depression among the elderly. Nonetheless, further research is needed on the subject, with stricter methodological approaches.

Keywords: Systematic review. Epidemiology. Elderly people. Depression. Physical activity. Exercise.

\section{Introdução}

Considerados talvez as maiores causas de sofrimento emocional e piora da qualidade de vida, a depressão e os transtornos depressivos são alterações que acontecem com bastante freqüência na população como um todo e, especialmente, entre os idosos ${ }^{1}$, constituindo um problema de grande magnitude para a saúde pública. Entretanto, parece não haver consenso na literatura quanto aos seus índices de prevalência ${ }^{2}$ : diferentes autores sugeriram valores de um a $10 \%$ entre os idosos vivendo na comunidade 3 , e 10 a $30 \%$ entre aqueles institucionalizados ${ }^{4}$.

Embora as doenças mentais não apresentem taxas muito elevadas de mortalidade, possuem, todavia, um grande peso de incapacidade e duração longa ${ }^{5}$ Idosos deprimidos de uma coorte na China exibiram maior risco relativo de apresentarem piora da função física e incapacidade funcional, sobretudo naqueles itens de atividades instrumentais da vida diária (AIVDs) relacionados à atividade física ${ }^{6}$. Achados semelhantes foram encontrados por CroninStubbs et al. ${ }^{7}$, que identificaram uma maior propensão à incapacidade funcional em idosos com sintomas depressivos leves.

A depressão em idosos é subdiagnosticada e subtratada ${ }^{8}$. Nos idosos, o diagnóstico é dificultado por problemas físicos coexistentes e, freqüentemente, os médicos encaram os sintomas depressivos como manifestações normais do envelhecimento e não tomam as atitudes necessárias ${ }^{9}$. Entretanto, a depressão é um problema de saúde comum e tratável: de 80 a $90 \%$ dos que sofrem desse transtorno podem ser efetivamente tratados e quase todos que recebem tratamento apresentam algum benefício ${ }^{10}$. Idosos deprimidos e não tratados têm mais incapacidades, uso aumentado dos serviços de saúde e morte prematura $^{2}$.

Nenhuma teoria explica satisfatoriamente a etiologia da depressão no idoso. Alterações no sistema nervoso central (SNC) (diminuição do número de neurô- 
nios e de neurotransmissores) e uma maior susceptibilidade biológica são possíveis explicações, além do fato de o idoso se submeter obrigatoriamente a mais estressores psicológicos e sociais do que qualquer outra faixa etária, tais como empobrecimento financeiro, declínio social, perda de papéis produtivos, solidão e perda de pessoas queridas. Além disso, a deterioração da capacidade física e mental restringe sua capacidade de interagir com o ambiente, reforçando o isolamento. A distinção entre os fatores biológicos, psicológicos e sociais é importante, mas a depressão no idoso parece ser o produto heterogêneo da interação entre diversos fatores predisponentes e desencadeantes, em proporções variáveis ${ }^{3}$.

Tem sido demonstrada na literatura uma associação positiva entre níveis elevados de atividade física e boa saúde mental ${ }^{11-}$ ${ }^{13}$. A prática de atividades físicas entre os idosos favorece a interação social, melhora a auto-eficácia (crença do indivíduo na sua capacidade de desempenho em atividades específicas) e proporciona uma maior sensação de controle sobre os eventos e demandas do meio ${ }^{14}$. O efeito benéfico do exercício físico em idosos deprimidos reside em uma série de fatores: melhora do humor, redução das respostas fisiológicas ao estresse, efeitos positivos na imagem corporal, no funcionamento cognitivo e na auto-estima, além de melhora na qualidade do sono e maior satisfação com a vida ${ }^{12,14}$. Mecanismos neuro-endócrinos são também apontados como responsáveis pelos efeitos antidepressivos no humor, tais como alterações na atividade central de norepinefrina e aumento da secreção de serotonina e endorfina ${ }^{12,15}$. Seja com vistas à prevenção primária ${ }^{1,10,12,14,16}$ ou secundária $^{13,15,17-19}$, o exercício físico tem sido estudado como forma de tratamento da depressão em diferentes populações de idosos.

Os idosos são geralmente mais sensíveis aos efeitos colaterais dos medicamentos antidepressivos e apresentam, com alguma freqüência, doenças médicas associadas e fazem uso de outras medicações, o que aumenta o risco de ocorrerem complicações clínicas e interações medicamentosas ${ }^{20}$. Foi também demonstrado que o uso de remédios para ansiedade, depressão ou insônia por idosos da comunidade estava associado a uma pior qualidade de vida desses indivíduos ${ }^{21}$. Nesse sentido, a atividade física demonstra-se vantajosa no tratamento da depressão, uma vez que não apresenta os efeitos colaterais e adversos da farmacoterapia, não custa caro e proporciona uma série de outros benefícios à saúde física da pessoa idosa, prevenindo seu declínio físico e funcional. Vários estudos têm demonstrado que a aderência a um programa regular de exercícios pode melhorar significativamente a força muscular, o tempo de reação, o controle do equilíbrio e a velocidade da marcha, assim como o desempenho cognitivo do idoso ${ }^{19}$.

Tendo em vista a dor e o sofrimento provocados por essa doença e o impacto negativo na qualidade de vida dessas pessoas, além dos custos elevados gerados pelos cuidados com a saúde mental da população, é oportuno e de grande relevância buscar na literatura os achados das pesquisas que investigaram o exercício físico como uma intervenção viável, acessível, barata e não-farmacológica, mas ainda pouco utilizada no âmbito da prevenção da depressão em idosos. Sendo assim, o objetivo deste trabalho é realizar uma revisão sistemática dos estudos epidemiológicos que analisaram o papel da atividade física na prevenção primária e secundária da depressão em pessoas idosas.

\section{Material e Métodos}

Trata-se de uma revisão sistemática de trabalhos científicos que estudaram a relação entre os transtornos depressivos (depressão maior, depressão menor e distimia) em pessoas idosas e sua associação com a atividade física, seja através do nível de influência dessa prática no desfecho da doença, ou utilizando a atividade física como uma das variáveis estudadas. A identificação dos artigos foi feita através de 
busca bibliográfica na base de dados MEDLINE, referente aos anos de 1995 a 2004. A estratégia de busca utilizada foi: (late life depression AND exercise) OR (late life depression AND physical exercise) OR (late life depression AND physical activity) OR (geriatric depression AND exercise) OR (geriatric depression AND physical exercise) OR (geriatric depression AND physical activity) OR (major depressive disorder in older persons AND exercise) OR (major depressive disorder in older persons AND physical exercise) OR (major depressive disorder in older persons AND physical activity). A biblioteca virtual SciELO foi também utilizada, buscando-se as seguintes palavras-chaves: (elderly AND depression) OR (idosos $\mathrm{E}$ depressão), referente ao mesmo período. Outra estratégia utilizada foi a busca manual em listas de referências dos artigos identificados e selecionados. A busca foi conduzida em janeiro de 2005. A seleção dos artigos baseou-se na conformidade dos limites dos assuntos aos objetivos deste trabalho, tendo sido desconsiderados aqueles que, apesar de aparecerem no resultado da busca, não abordavam o assunto sob o ponto de vista da epidemiologia da depressão no idoso e da atividade física. Foram considerados critérios de inclusão os estudos de caso-controle, transversais, longitudinais, ensaios clínicos/estudos controladosrandomizados que tenham sido publicados em português, inglês ou espanhol, sendo excluídos os estudos publicados nos demais idiomas.

\section{Resultados}

Seguindo a estratégia definida, a busca bibliográfica resultou em 191 artigos (150 da base MEDLINE, 21 da biblioteca SCIELO e outros 20 estudos identificados nas referências bibliográficas desses trabalhos). De acordo com os objetivos do estudo e critérios de inclusão, 16 artigos foram selecionados, sendo que os estudos de Babyac et al. ${ }^{13}$ e Blumenthal et al. ${ }^{15}$ foram analisados conjuntamente por terem estudado a mesma amostra, resultando então num total de 15 trabalhos. Os países onde os estudos foram desenvolvidos estão assim distribuídos: EUA ( $\mathrm{n}=7 ; 46,6 \%)$, Holanda $(n=2 ; 13,3 \%)$, Brasil $(n=2 ; 13,3 \%)$, Inglaterra ( $\mathrm{n}=1 ; 6,7 \%)$, Japão $(\mathrm{n}=1 ; 6,7 \%)$, Colômbia $(n=1 ; 6,7 \%)$ e Finlândia $(n=1$; $6,7 \%)$. No que diz respeito à idade da amostra, embora a Organização Mundial de Saúde defina para os idosos a faixa etária de 65 anos ou mais em países desenvolvidos e 60 anos ou mais em países em desenvolvimento ${ }^{22}$, nem todos os estudos seguiram exclusivamente essa faixa de idade em suas amostras. Apesar de todas as amostras possuírem idosos em sua composição, apenas $53,3 \%(n=8)^{8,10,14,18,19,23-25}$ delas eram exclusivas de pessoas com 60 anos ou mais.

Para melhor compreensão e visualização dos resultados da busca bibliográfica, os trabalhos foram agrupados de acordo com o delineamento metodológico utilizado. Do total de 15 estudos, oito foram de intervenção e sete foram observacionais. A Tabela 1 apresenta as características dos estudos selecionados segundo autor, ano de realização, tipo de estudo, país de origem, amostra (tamanho, perdas, idade e sexo), tempo de duração do estudo e ajuste das variáveis de confundimento. Foram encontrados oito estudos de intervenção (sendo todos eles do tipo controlados-randomizados) e sete de observação (dos quais cinco transversais e dois de coorte prospectivo). Dos estudos controlados-randomizados, cinco $(62,5 \%)$ foram realizados nos EUA, um $(12,5 \%)$ na Inglaterra, um (12,5\%) na Holanda e um $(12,5 \%)$ no Brasil. Dos estudos transversais, um (20\%) foi desenvolvido na Finlândia, um (20\%) nos EUA, um (20\%) na Colômbia, um (20\%) no Japão e outro (20\%) no Brasil. Dentre os estudos de coorte, um (50\%) foi realizado nos EUA e o outro (50\%), na Holanda.

Muitos estudos sugerem uma relação de associação entre a prática de atividades físicas e a depressão em idosos. No entanto, a natureza e a força da associação vari- 
Tabela 1 - Características metodológicas dos estudos selecionados sobre influência da atividade física no estado de depressão de pessoas idosas, 1995-2004.

Table 1 - Methodological features of selected studies on the influence of exercise on depressed states in the elderly, 19952004.

\begin{tabular}{|c|c|c|c|c|c|}
\hline Autor/Ano & $\begin{array}{l}\text { Tipo do } \\
\text { estudo/País }\end{array}$ & $\begin{array}{l}\text { Amostra/ } \\
\text { Perdas }\end{array}$ & Idade/Sexo & $\begin{array}{l}\text { Tempo de } \\
\text { duração do } \\
\text { estudo }\end{array}$ & $\begin{array}{l}\text { Variáveis } \\
\text { de ajuste }\end{array}$ \\
\hline \multicolumn{6}{|l|}{ Estudos de Intervenção } \\
\hline $\begin{array}{l}\text { Blumenthal et al., } \\
1999^{18} \text {; e }\end{array}$ & CR / EUA & $156 / 0,5 \%$ & 5-77 / Ambos & $\begin{array}{l}4 \text { meses } \\
\text { (intervenção) }\end{array}$ & Não \\
\hline Babyac et al., $2000^{16}$ & CR / EUA & $156 / 4,7 \%$ & $\begin{array}{l}550 \text { ou mais / } \\
\text { Não mencionado }\end{array}$ & $\begin{array}{l}10 \mathrm{~m} \text { (4 int }+6 \\
\text { acompanhamento) }\end{array}$ & $\operatorname{Sim}(1,2,3$ e 4$)$ \\
\hline Singh et al., $2001^{21}$ & CR / EUA & $32 / 6 \%$ & $\begin{array}{l}\text { 69-73 (média: } \\
\text { 71,3) / Ambos }\end{array}$ & $\begin{array}{l}26 \text { semanas } \\
20 \text { int }+6 \text { acomp) }\end{array}$ & Não \\
\hline Mather et al., $2002^{14}$ & $\mathrm{CR} /$ Inglaterra & $86 / 1,2 \%$ & $\begin{array}{l}53-91(64) / \\
\text { Ambos }\end{array}$ & $\begin{array}{l}34 \text { s (10 int }+ \\
24 \text { acomp) }\end{array}$ & Não \\
\hline Teri et al., $2003^{20}$ & CR / EUA & $153 / 1,8 \%$ & $\begin{array}{l}55-93(78) / \\
\text { Ambos }\end{array}$ & $\begin{array}{l}24 \mathrm{~m} \text { (3 int }+ \\
21 \text { acomp) }\end{array}$ & Não \\
\hline $\begin{array}{l}\text { Cicchanowski et al., } \\
2004^{10}\end{array}$ & CR / EUA & $138 / 0$ & $\begin{array}{l}60 \text { ou }+(73) / \\
\text { Ambos }\end{array}$ & $12 \mathrm{~m}$ (int) & $\operatorname{Sim}(2,3,5$ e 6$)$ \\
\hline Paw et al., $2004^{28}$ & CR / Holanda & $224 / 2,8 \%$ & $\begin{array}{l}\text { 64-94 (81) / } \\
\text { Ambos }\end{array}$ & $6 \mathrm{~m}$ (int) & $\operatorname{Sim}(2,3$ e 7$)$ \\
\hline Ersek et al., $2004^{26}$ & CR / EUA & $273 / 9,8 \%$ & $\begin{array}{l}65 \text { ou }+/ \\
\text { Não menc }\end{array}$ & $\begin{array}{l}48 \text { s (7 int }+ \\
41 \text { acomp) }\end{array}$ & Não menc \\
\hline $\begin{array}{l}\text { Bastone e Jacob Filho, } \\
2004^{22}\end{array}$ & CR / Brasil & $40 / 7,5 \%$ & $\begin{array}{l}60-99(78,5) / \\
\text { Ambos }\end{array}$ & $6 \mathrm{~m}$ (int) & $\operatorname{Sim}(2,8$ e 9$)$ \\
\hline \multicolumn{6}{|l|}{ Estudos de Observação } \\
\hline $\begin{array}{l}\text { Ruuskanen e } \\
\text { Ruoppila, } 1995^{17}\end{array}$ & TR / Finlândia & $1244 / 22,2 \%$ & $\begin{array}{l}\text { 65-84 / } \\
\text { Não menc }\end{array}$ & $12 \mathrm{~m}$ & Não \\
\hline $\begin{array}{l}\text { Kritz-Silverstein } \\
\text { et al., } 2001^{15}\end{array}$ & TR / EUA & 944 / 53,5\% & $\begin{array}{l}\text { 50-89 / } \\
\text { Ambos }\end{array}$ & $\begin{array}{l}6 \text { anos }(2 \text { cortes } \\
\text { tranv de } 3 \text { a: } \\
84-87 / 92-95)\end{array}$ & $\begin{array}{l}\operatorname{Sim}(2,10,11 \\
12,13,14 \text { e } 15)\end{array}$ \\
\hline $\begin{array}{l}\text { Strawbridge } \\
\text { et al., } 2002^{29}\end{array}$ & CO PR / EUA & 1947 / 8,3\% & $\begin{array}{l}\text { 50-94 (63) / } \\
\text { Ambos }\end{array}$ & 5 a (94-99) & $\begin{array}{l}\operatorname{Sim}(2,3,9,10,11, \\
12,13,16,17 \text { e } 18)\end{array}$ \\
\hline $\begin{array}{l}\text { Tuesca-Molina } \\
\text { et al., } 2003^{12}\end{array}$ & TR / Colômbia & $602 / 7,8 \%$ & $\begin{array}{l}\text { 60-94 / } \\
\text { Ambos }\end{array}$ & $12 \mathrm{~m}$ & Não \\
\hline $\begin{array}{l}\text { Van Gool et al., } \\
2003^{30}\end{array}$ & $\begin{array}{l}\text { CO PR / } \\
\text { Holanda }\end{array}$ & $1280 / 58,8 \%$ & $\begin{array}{l}\text { 55-85 / } \\
\text { Ambos }\end{array}$ & 6 a (92-93/98-99) & $\begin{array}{l}\operatorname{Sim}(2,3,18 \mathrm{e} \\
19)\end{array}$ \\
\hline $\begin{array}{l}\text { Fukukawa } \\
\text { et al., } 2004^{19}\end{array}$ & $\begin{array}{l}\text { TR (cross-lagged } \\
\text { panel design) / } \\
\text { Japão }\end{array}$ & $1151 / 0,3 \%$ & $\begin{array}{l}40-79(57,4) / \\
\text { Ambos }\end{array}$ & $2 \mathrm{a}(97-00 / 00-02)$ & $\operatorname{Sim}(3,9$ e 17$)$ \\
\hline $\begin{array}{l}\text { Gazalle et al., } \\
2004^{27}\end{array}$ & TR / Brasil & $556 / 4,7 \%$ & $\begin{array}{l}60 \mathrm{ou}+1 \\
\text { Ambos }\end{array}$ & $3 \mathrm{~m}$ & $\begin{array}{l}\operatorname{Sim}(2,3,11,9 \\
20,21,22 \text { e } 23)\end{array}$ \\
\hline
\end{tabular}

Tipo de estudo (Study design): CR - controlado-randomizado (randomized-controlled trial); TR - transversal (cross-sectional); CO PR - coorte prospectivo (prospective cohort) / Variáveis de ajuste (Control variables): 1 - nível de depressão na linha de base (baseline level of depression); 2 - idade (age); 3 - sexo (gender); 4 - uso de medicamentos antidepressivos durante o seguimento (use of antidepressant drugs during the follow-up); 5 - nível de neuroticismo (level of neuroticism); 6 - nível de distimia (level of dysthymia); 7 - freqüência às sessões de treinamento (class attendance); 8 - tempo de institucionalização (length of hospitalization); 9 - presença de doenças crônicas (presence of chronic diseases); 10 - índice de massa corporal (body mass index); 11 - tabagismo (smoking); 12 uso de álcool (alcohol consumption); 13 - suporte social (social support); 14 - situação conjugal (marital status); 15 - uso de estrogênio (use of estrogen); 16 - raça (ethnicity); 17 - renda (income); 18 - presença de incapacidades (physical disability); 19 - escolaridade (schooling); 20 - nível social (social status); 21 - trabalho remunerado (employment); 22 - morte de pessoa importante no último ano (death of significant other uring the past year); 23 - baixa atividade física (low level of physical activity). 
aram nas diferentes amostras, bem como o tipo de intervenção/observação e o instrumento usado para medir a variável dependente. A Tabela 2 apresenta as características dos estudos selecionados.

De acordo com a Tabela 2, dos oito estudos controlados-randomizados, um utilizou um programa de exercícios aeróbios como variável de intervenção ${ }^{13,15}$, outro utilizou sessões de exercícios de força (treinamento com pesos $)^{18}$, quatro se basearam em ambos (aeróbios e de força) $)^{11,17,19,25} \mathrm{e}$ dois não especificaram o tipo de exercício proposto $^{8,23}$. A mesma tabela mostra ainda uma certa variedade entre os estudos de observação quanto às formas de obtenção da variável independente (atividade física) nas amostras analisadas: um deles (coorte) quantificava o nível de atividades físicas (domésticas e esportivas) de acordo com a intensidade (leve ou pesada) e o tempo gasto em minutos/ $\mathrm{dia}^{27}$; outro (transversal), quantificava a distância caminhada por dia através do número mé-

Tabela 2 - Características dos estudos selecionados segundo autor/ano, tipo de intervenção/observação, instrumento utilizado para medir a depressão, tipo de associação encontrada e p-valor.

Table 2 - Features of studies selected according to author/year, type of intervention/observation, instrument used to evaluate depression, kind of association found and p-value.

\begin{tabular}{|c|c|c|c|c|}
\hline Autor / ano & Tipo de interv./observ. & $\begin{array}{l}\text { Instrum. de } \\
\text { medida da dep. }\end{array}$ & $\begin{array}{l}\text { Tipo da } \\
\text { associação* }\end{array}$ & P-valor \\
\hline \multicolumn{5}{|l|}{ Estudos de Intervenção } \\
\hline Blumenthal et al., $1999^{18}$; e & Sessões de exercícios aeróbios & $H D R S ; B D I$ & + & $<0.001$ \\
\hline Babyac et al., $2000^{16}$ & Sessões de ex aer & $H D R S ; B D I$ & + & 0.0009 \\
\hline Singh et al., $2001^{21}$ & Sessões de ex de força & $B D I$ & + & 0.047 \\
\hline Mather et al., $2002^{14}$ & $\begin{array}{l}\text { Sessões de ex aer, de } f \\
\text { e flexibilidade }\end{array}$ & HDRS; GDS & + & 0.05 \\
\hline Teri et al., $2003^{20}$ & $\begin{array}{l}\text { Sessões de ex aer, de f, } \\
\text { flex e equilíbrio }\end{array}$ & $H D R S ; C S D$ & + & 0.02 \\
\hline Cicchanowski et al., $2004^{10}$ & Não menc & $D H S C L-20$ & + & 0.002 \\
\hline Paw et al., $2004^{28}$ & Sessões de ex aer e de $f$ & GDS & 0 & Não menc \\
\hline Ersek et al., $2004^{26}$ & Não menc & GDS & Não menc & Não menc \\
\hline Bastone e Jacob Filho, $2004^{22}$ & Sessões de ex aer e de $f$ & GDS & + & 0.000 \\
\hline \multicolumn{5}{|l|}{ Estudos de Observação } \\
\hline Ruuskanen e Ruoppila, $1995^{17}$ & $\begin{array}{l}7 \text { Atividade física medida } \\
\text { subjetivamente }\end{array}$ & $B D I$ & + & Não menc \\
\hline Kritz-Silverstein et al., $2001^{15}$ & $\begin{array}{l}\text { Af medida subjetivam pela } \\
\text { prática durante o mês anterior }\end{array}$ & $B D I$ & 0 & $>0.1$ \\
\hline Strawbridge et al., $2002^{29}$ & $\begin{array}{l}\text { Af medida subjetivam por escala } \\
\text { construída pelos autores }\end{array}$ & $D S M-12 D$ & + & Não menc \\
\hline Tuesca-Molina et al., $2003^{12}$ & $\begin{array}{l}\text { Af medida pela participação em } \\
\text { grupos de socialização }\end{array}$ & HDRS & + & 0.001 \\
\hline Van Gool et al., $2003^{30}$ & $\begin{array}{l}\text { Af medida subjetivam em minutos } \\
\text { praticados/dia }\end{array}$ & $C E S-D$ & + & 0.038 \\
\hline $\begin{array}{l}\text { Fukukawa et al., } 2004^{19} \\
\text { caminhada/dia }\end{array}$ & Af medida por distância & $C E S-D$ & + & $<0.05$ \\
\hline Gazalle et al., $2004^{27}$ & $\begin{array}{l}\text { Af medida subjetivam por } \\
\text { escala padronizada (IPAQ) }\end{array}$ & $\begin{array}{l}\text { Questionário idealizado } \\
\text { pelos autores }\end{array}$ & 0 & 0.2 \\
\hline
\end{tabular}

*Optou-se por exibir o tipo da associação dessa forma (positiva, negativa ou nula) uma vez que as medidas de associação utilizadas foram variadas entre os diferentes trabalhos, interferindo na sua comparabilidade. IPAQ - International Physical Activity Questionnaire (versão reduzida/brief version); HDRS - Hamilton Depression Rating Scale; BDI - Beck Depression Inventory; GDS - Geriatric Depression Scale; CSDD - Cornell Scale for Depression in Dementia; HSCL-20 - Hopkins Symptom Checklist (versão reduzida/ brief version); DSM-12D - Diagnostic and Statistical Manual of Mental Disorders, F.E.; CES-D - Center for Epidemiologic Studies Depression Scale. 
dio de passos durante uma semana, monitorados por um pedômetro ${ }^{16}$; dois estudos (um transversal e outro de coorte) determinavam a freqüência usual de exercícios físicos de esporte e lazer através de pontuação obtida numa escala ${ }^{24,26}$; um outro (transversal) avaliava o nível de atividade física pela participação em atividades recreativas de grupos de socialização ${ }^{10}$; e por fim, outros dois estudos (transversais) faziam uma avaliação subjetiva da freqüência e intensidade das atividades físicas através de entrevista estruturada ${ }^{14}$ e padronizada $^{12}$. Este último buscava ainda validar as informações pela correlação com valores de freqüência cardíaca e níveis de colesterol sérico da amostra.

\section{Discussão}

No que diz respeito à metodologia adotada nos diferentes trabalhos, vários aspectos referentes aos desenhos utilizados podem gerar limitações que afetam a acurácia e a validade das estimativas inferidas. Os mais comuns foram: uso de amostras reduzidas, curtos períodos de seguimento, ausência de acompanhamento após o término do tratamento (no sentido de investigar possíveis recaídas), perdas de seguimento, não-ajustamento das variáveis de confundimento e ausência de um "grupo controle” (grupo de referência) estruturado que permitisse analisar o efeito da intervenção na ausência de contato social entre os sujeitos das amostras. Além disso, encontrou-se grande variação de faixas etárias nas amostras estudadas e nem todos os autores seguiram parâmetros e definições internacionais como critérios de inclusão, dificultando a comparabilidade entre os estudos. Outros critérios utilizados podem levar à ocorrência de erros sistemáticos (vieses), como o fato das informações serem obtidas por meio de estratégias recordatórias de pessoas idosas e baseadas em percepções subjetivas do grupo avaliado (viés de informação), da seleção da amostra ser realizada através de anúncios em meios de comunicação como jornais e revistas (viés de seleção), e de terem sido usados instrumentos de medida da depressão válidos para a população em geral e não específicos para idosos (viés de aferição).

O uso de amostras reduzidas implica em estudar um grupo de pessoas que não seja representativo do domínio de interesse. Dos estudos de intervenção analisados, a maior amostra era representada por 273 indivíduos ${ }^{23}$, enquanto a menor era composta por 32 sujeitos $^{18}$ (média $=139,8 /$ desvio padrão $=79,1$; mediana $=153$ ). Entre os estudos observacionais investigados, a maior amostra era de 1.947 indivíduos ${ }^{26} \mathrm{e}$ a menor igual a $556^{24}$ (média $=1103,4 /$ d.p. $=472,8$; mediana $=1.151$ ). No que diz respeito às diferenças de proporção entre homens e mulheres nas amostras, dois es$\operatorname{tudos}^{13,15,23}$, ou $13,3 \%$ do total, não forneceram essa informação, mas entre os que o fizeram, foi observada uma maior presença do sexo feminino; somente dois estudos $^{16,17}$ apresentaram predominância de homens entre os sujeitos investigados. Esse fato pode ser entendido como uma situação “esperada”, uma vez que as mulheres representam maioria na população de idosos como um todo, e a maior parte dos trabalhos científicos que envolvem esse grupo etário tem geralmente uma participação mais forte do sexo feminino. Entretanto, a maior presença de mulheres nos trabalhos analisados pode, de alguma maneira, indicar uma maior prevalência de depressão em idosas, contrariando os dados sugeridos pela literatura de que após os 65 anos os episódios de depressão são mais freqüentes entre os homens. Dos estudos de intervenção analisados, 50\% utilizaram a presença de depressão maior ou menor, distimia ou sintomas depressivos como critério de inclusão ${ }^{8,11,13,15,18}$ e todos eles tinham as mulheres como maioria em suas amostras. De todos os estudos de observação investigados, a maior parte dos sujeitos que já apresentavam depressão na linha de base ou que desenvolveram a doença no seguimento era do sexo feminino. Em um deles ${ }^{24}$, os autores apontam a mai- 
or média de sintomas depressivos no sexo feminino como um achado especialmente relevante porque que refuta a idéia de que a depressão é mais diagnosticada em mulheres, em função de procurarem mais os serviços de saúde, uma vez que o estudo foi realizado em domicílios da comunidade. Tuesca-Molina et al. ${ }^{10}$ também encontraram uma maior prevalência de depressão entre as mulheres da amostra e sugerem como uma possível explicação o fato da mulher assumir uma carga de responsabilidades elevada, dada a sua condição de esposa, mãe, educadora e cuidadora de outras pessoas. Para Almeida ${ }^{28}$, outros fatores que possivelmente contribuem para que as mulheres sejam mais vulneráveis ao desenvolvimento de transtornos mentais na velhice incluem alta taxa de viuvez, isolamento social e privação de estrogênio.

Foram encontrados períodos de seguimento variados entre os trabalhos analisados. A depressão, por se tratar de uma doença crônica, torna o indivíduo suscetível a recaídas; daí a importância de continuar a avaliá-lo após o fim do tratamento, mesmo após a remissão dos sintomas da doença. De acordo com a Tabela 1, quatro estudos de intervenção (50\%) não acompanharam a amostra após o final do tratamento com exercícios. A mesma tabela também apresenta o percentual de indivíduos perdidos durante a realização dos estudos. Perdas de seguimento acentuadas podem levar à distorção das estimativas de efeito, sobretudo quando estas ocorrem de forma seletiva entre os grupos da amostra, pois fatores relacionados à exposição podem estar influenciando a participação dos indivíduos no estudo, com isso comprometendo sua validade. Seis dos oito estudos controlados-randomizados ${ }^{11,13,15,18,19,25}$ analisaram a distribuição das perdas entre os grupos em estudo, sendo que dois deles $^{15,25}$ apresentaram ainda as características dos indivíduos que foram perdidos. Desses seis, dois ${ }^{13,15,25}$ exibiram tendência a um direcionamento das perdas: enquanto no primeiro estas foram concentradas no grupo que sofreu a intervenção com exercícios, no outro isso ocorreu no grupo que não se exercitou. As perdas de seguimento diferenciais constituem um importante viés de seleção também no contexto dos estudos de coorte, uma vez que os indivíduos perdidos no seguimento tendem a ter diferentes probabilidades de apresentar o desfecho em questão, em comparação com os que permanecem no estudo, produzindo falsas estimativas de incidência; nesse caso, é possível comparar as características da linha de base relacionadas ao desfecho de interesse entre indivíduos perdidos e mantidos na observação ${ }^{29}$. Dois estudos observacionais ${ }^{14,27}$ caracterizaram as perdas de seguimento. Van Gool et al. ${ }^{27}$ apontaram como limitação do seu estudo o fato da maior parte das perdas sofridas pela coorte apresentar características semelhantes (homens, mais velhos e com mais doenças crônicas), pondo em risco a capacidade de generalização de seus resultados. Para Reichenheim e Moraes ${ }^{30}$, a validade de seguimento em estudos longitudinais estará assegurada quando o risco de perda durante o período de acompanhamento não estiver associado ao desfecho.

Um procedimento comum nos trabalhos analisados diz respeito ao tipo de instrumento utilizado para avaliar o nível de depressão das amostras. De acordo com a Tabela 2, vê-se que a maioria dos estu$\operatorname{dos}^{8,10,12-16,18,26,27}$ ( $n=10$, ou 62,5\%) fez o uso de escalas de depressão válidas para a população em geral, mas não específicas para pessoas idosas. Idosos têm características cognitivas peculiares e a depressão entre esses indivíduos apresenta manifestações clínicas diferentes do adulto jovem. Nesse sentido, os instrumentos válidos para a população em geral não contemplariam essas diferenças, pois foram construídos para avaliar os sintomas mais característicos da depressão em adultos. Gazalle et al. ${ }^{24}$ vão além, argumentando que o subdiagnóstico da depressão no idoso poderia ser reforçado pelo uso de instrumentos inadequados às pessoas mais velhas. Outro fator capaz de introduzir o viés de informação foi dado 
pela forma de coleta da variável independente: os níveis de atividade física das amostras foram, em geral, coletados de forma retrospectiva e por isso sujeitos ao viés de memória, sobretudo em se tratando de pessoas idosas e, portanto, mais suscetíveis a esse problema.

Fatores de confundimento podem influenciar o estado de depressão ao final do seguimento (desfecho) e estar associados ao nível usual de atividades físicas dos sujeitos da amostra, bem como à sua adesão ao programa de exercícios proposto (exposição). Nesse sentido, o não ajustamento de variáveis como, por exemplo, idade, sexo, renda, hábitos de vida ou condições crônicas de saúde, poderia levar a associações espúrias. Metade dos estudos de intervenção $(n=4)$ controlaram as variáveis de confundimento ${ }^{8,13,19,25}$, enquanto $71,4 \%$ dos estudos observacionais $(n=5)^{12,16,24,26,27}$ também adotaram esse procedimento.

Um outro fator que também poderia viciar o estudo diz respeito à seleção da amostra: dos 15 trabalhos avaliados, sete estudos controlados-randomiza$\operatorname{dos}^{8,11,13,15,17,18,23,25}$ recrutaram os sujeitos da amostra de instituições de longa permanência (long-term care facilities), comunidades de aposentados (retirement communities), residências públicas para idosos (senior public housing), ou da comunidade, por meio de panfletos, anúncios diretos em jornais e rádios locais, cartas enviadas a médicos de unidades de saúde mental, ou enviadas aos próprios indivíduos a partir de listas de pacientes de unidades de cuidados primários ou de serviços psiquiátricos. Sendo assim, as amostras eram compostas de idosos que se apresentavam voluntariamente e que, a princípio, tinham interesse em participar de um estudo envolvendo o treinamento com exercícios como tratamento de depressão. Pode-se presumir que muitos desses participantes acreditavam na atividade física como uma terapia favorável contra a depressão e poderiam estar, assim, mais "inclinados" a crer na eficácia desse tipo de tratamento - resultando na sele- ção de amostra viciada. Um estudo controlado-randomizado ${ }^{19}$ obteve sua amostra selecionando idosos residentes em casas geriátricas (nursing homes) através de entrevista e incluindo-os de acordo com os critérios de elegibilidade do estudo. Dos estudos observacionais, $57,1 \%(n=5)^{14,16,24,26}$ referiram a seleção das amostras por meio de processos de amostragem probabilística, sendo os idosos, em sua maioria, residentes da comunidade.

Um aspecto importante observado entre os trabalhos analisados reside na possibilidade da interação social ocorrida entre os participantes dos estudos representar um fator de confusão: não se sabe até que ponto o bem-estar psicológico causado pela atividade física pode ser devido ao exercício em si ou às redes de relações afetivas proporcionadas por sua prática. Dos trabalhos analisados, $46,7 \%$ (cinco estudos de intervenção ${ }^{11,13,15,18,19,25}$ e dois de observação ${ }^{12,26}$ ) fizeram menção a essa questão. Efeitos como distração, divertimento e, sobretudo, a sensação de pertencimento a um grupo, são aspectos importantes na vida de indivíduos para os quais a solidão e o isolamento estão presentes em seu dia-a-dia. Embora o fator social possa ser considerado inerente à prática de exercícios, alguns estudos propõem alternativas no sentido de controlar essa variável. Mather et al. ${ }^{11}$ sugeriram o uso de um grupo controle estruturado, no qual os exercícios propostos fossem realizados individualmente. Assim, os efeitos isolados da atividade física poderiam ser mais bem discriminados e dissociados dos efeitos psicossociais da intervenção em grupo. Uma outra possibilidade utilizada foi proposta por Paw et al. ${ }^{25}$, que usaram como controle um grupo de indivíduos que não se exercitou, mas assistiu a palestras e participou de discussões sobre temas de interesse dos idosos. Nesse caso, "casos" e "controles" eram expostos ao contato social, sendo que o exercício físico era praticado somente pelos casos. Além disso, dois trabalhos ${ }^{12,26}$ controlaram o nível de suporte social dos sujeitos da amostra atra- 
vés do ajustamento dessa variável em suas análises.

Um último aspecto a ser considerado consiste na direção da associação estabelecida entre atividade física e depressão. Alguns estudos sugerem a possibilidade de causalidade recíproca na relação entre essas duas variáveis, embora isso possa ser resultado do tipo de delineamento utilizado em cada estudo, que impedia o estabelecimento de uma relação causal entre a exposição e o desfecho devido à ausência do fator temporal. Um estudo transversal ${ }^{14}$ concluiu que a atividade física pode ter efeitos benéficos no bem-estar psicológico dos idosos da amostra, e vice-versa. De acordo com os achados de outro estudo transversal ${ }^{12}$, tanto o exercício leva a uma menor incidência de humor deprimido, quanto a presença de humor deprimido leva à menor prática de exercícios. Van Gool et al. ${ }^{27}$ acompanharam uma coorte de idosos e concluíram que a depressão é um fator de risco para o sedentarismo: pessoas que desenvolveram depressão tornaram-se sedentárias com maior freqüência, enquanto aqueles indivíduos que tiveram seu quadro depressivo remitido passaram a se exercitar com maior regularidade. Um único estudo controlado-randomizado ${ }^{13}$ apontou esse tipo de relação recíproca entre atividade física e estado de humor deprimido, sugerindo que sentir-se menos deprimido pode tornar o indivíduo mais propenso a se manter fisicamente ativo, $\mathrm{e}$ continuar a se exercitar torna menos provável o retorno dos sintomas depressivos.

\section{Conclusão}

A literatura científica parece, de maneira geral, sustentar os efeitos benéficos da atividade física e do exercício na depressão em pessoas idosas. Apesar disso, não há dúvidas quanto à necessidade de mais pesquisas sobre o assunto e que apresentem um maior rigor metodológico. Sugere-se o uso de amostras maiores e mais representativas do denominador em questão, ajustamento das variáveis de confun- dimento, uso de instrumentos de medida da depressão validados para a população idosa e, no caso dos estudos experimentais, o acompanhamento da amostra após o término da intervenção (buscando identificar possíveis recaídas). Adicionalmente, propõe-se a utilização de desenhos de estudo que captem a variável independente de forma prospectiva, assegurando análises livres do viés de memória.

Um tipo de confundimento particularmente freqüente nos estudos foi causado pelo contato social existente durante a prática de atividades físicas. Assim, propõese que sejam criadas estratégias buscando uma possível dissociação dos efeitos psicossociais do exercício daqueles puramente físicos. Além disso, alguns autores apontaram para a possibilidade de causalidade recíproca entre atividade física e depressão; nesse sentido, o desenvolvimento de estudos com tempos de seguimento mais longos poderia constituir um artifício válido visando uma identificação mais precisa da direção da relação causal.

Quanto aos índices de prevalência da depressão em idosos, sugerem-se estudos que esclareçam a maior ocorrência entre homens ou mulheres, bem como entre idosos mais jovens ou entre aqueles de idades mais avançadas, uma vez que esses dados foram confusos na literatura pesquisada. Além disso, são necessários estudos adicionais para determinar com maior grau de acurácia as características dos exercícios que seriam mais indicados no tratamento da depressão em idosos, como intensidade, duração, freqüência e tipo do exercício, tendo ainda em vista os diferentes níveis de manifestações da doença e, com isso, a obtenção de respostas diferenciadas de acordo com o tipo de exercício praticado.

No âmbito da saúde pública, a atividade física se apresenta como uma forma de tratamento acessível, barata, não-farmacológica e capaz de gerar nos idosos benefícios que excedem os efeitos antidepressivos, uma vez que promovem sua saúde mental e também física. Sendo assim, é importante que os profissionais de saúde 
estejam atentos e preparados para identificar e conduzir de maneira correta o tratamento dos pacientes idosos com depressão. Esses profissionais têm a função de modificar o paradigma atual, cuja tendência consiste em adotar ações que visam primordialmente à medicalização das manifestações de desequilíbrio da saúde mental. Isso não significa, no entanto, que um método seja melhor do que o outro: cada caso particular demandará condutas terapêuticas distintas. Por fim, é válido lembrar o contexto sociocultural onde a própria sociedade insere o idoso: a presença de estereótipos e preconceitos lhe impõem uma condição marginalizada, reforçando uma auto-imagem negativa e poten- cializando seu nível de estresse, colocando-o assim em maior risco para o desenvolvimento de distúrbios psíquicos.

Esta revisão apresentou como limitação o fato de não terem sido pesquisadas bases de dados diferentes de MEDLINE e da biblioteca SCIELO, o que reduz a identificação de estudos relevantes. No entanto, este problema foi minimizado ao se pesquisar as referências bibliográficas dos estudos selecionados. Embora quase todos os estudos tenham encontrado associações positivas entre atividade física e depressão, o viés de publicação, que decorre da não publicação de estudos com resultados negativos, também foi considerado uma possível limitação desta revisão.

\section{Referências}

1. Bird MJ, Parslow, LA. Potential for community programs to prevent depression in older people. Med J Aust 2002; 177(Suppl): S107-10.

2. Snowdon, J. How high is the prevalence of depression in old age? Rev Bras Psiquiatr 2002; 24(Supl I): 42-7.

3. Forlenza. OV. Fatores de Risco Associados à Morbidade Psiquiátrica em Idosos. In: Forlenza, OV, Almeida OP. Depressão e demência no idoso. São Paulo: Lemos Editorial; 1997.

4. Blazer DG. Depression in late life: review and commentary. J Gerontol A Biol Sci Med 2003; 58(3): 24965.

5. Schramm JMA, Oliveira AF, Leite IC, Valente JG, Gadelha AMJ, Portela MC, Campos MR. Transição epidemiológica e o estudo de carga de doença no Brasil. Ciência \& Saúde Coletiva 2004; 9(4): 897-908.

6. Jing J, Tang Z, Futatsuka M, Zhang K. Exploring the influence of depressive symptoms on physical disability: a cohort study of elderly in Beijing, China. Qual Life Res 2004; 13(7): 1337-46.

7. Cronin-Stubbs D, de Leon CF, Beckett LA, Field TS, Glynn RJ, Evans DA. Six-year effect of depressive symptoms on the course of physical disability in community-living older adults. Arch Intern Med 2000; 160(20): 3074-80.

8. Ciechanowski P, Wagner E, Schmaling K, Schwartz S, Williams B, Diehr P, Kulzer J, Gray S, Collier C, LoGerfo J. Community-integrated home-based depression treatment in older adults: a randomized controlled trial. JAMA 2004; 291(13): 1569-77.
9. Ministério da Saúde. Página eletrônica http:// portal.saude.gov.br/saude/visualizar _texto.cfm?idtxt=19108. Acessado em 20 de janeiro de 2005.

10. Tuesca-Molina R, Fierro HN, Molinares AS, Oviedo MF, Polo AY, Pólo CJ, Sierra MI. Los grupos de socializacion como factor protector contra la depresion em personas ancianas. Baranquilla, Colômbia. Rev Esp Salud Publica 2003; 77(5): 595-604

11. Mather AS, Rodriguez C, Guthrie MF, McHarg AM, Reid IC, McMurdo ME. Effects of exercise on depressive symptoms in older adults with poorly responsive depressive disorder: randomized controlled trial. $\mathrm{BrJ}$ Psychiatry. 2002; 180: 411-5.

12. Kritz-Silverstein D, Barret-Connor E, Corbeau C. Crosssectional and prospective study of exercise and depressed mood in the elderly: The Rancho Bernardo Study. Am J Epidemiol 2001; 153(6): 596-603.

13. Babyac MA, Blumenthal JA, Herman S, Khatri P, Doraiswamy M, Moore K, Craighead WE, Baldewicz TT, Krishnan KR. Exercise treatment for major depression: maintenance of therapeutic benefit at 10 months. Psychosom Med 2000; 62: 633-38.

14. Ruuskanen JM, Ruoppila I. Physical activity and psychological well-being among people aged 65 to 84 years. Age and Ageing 1995; 24(4): 292-6.

15. Blumenthal JA, Babyac MA, Moore KA, Craighead WE, Herman S, Khatri P, Waugh R, Napolitano MA, Forman LM, Appelbaum M, Doraiswamy PM, Krishnan KR. Effects of exercise training on older patients with major depression. Arch Intern Med 1999; 159(19): 2349-56. 
16. Fukukawa Y, Nakashima C, Tsuboi S, Kozakai R, Doyo W, Niino N, Ando F, Shimokata H. Age differences in the effect of physical activity on depressive symptoms. Psychol Aging 2004; 19(2): 356-51.

17. Teri L, Gibbons LE, McCurry SM, Logsdon RG, Buchner DM, Barlow WE, Kukull WA, LaCroix AZ, McCormick W, Larson EB. Exercise pus behavioral management in patients with Alzheimer disease: a randomized controlled trial. JAMA 2003; 290(15): 2015-22.

18. Singh NA, Clements KM, Singh MA. The efficacy of exercise as a long-term antidepressant in elderly subjects: a randomized, controlled trial. J Gerontol A Biol Sci Med Sci 2001; 56(8): M497-504.

19. Bastone AC, Filho WJ. Effect of an exercise program on functional performance of institutionalized elderly. $J$ Rehabil Res Dev 2004; 41(5): 659-68.

20. Scalco MZ. Tratamento de idosos com depressão utilizando tricíclicos, IMAO, ISRS e outros antidepressivos. Rev Bras Psiquiatr 2002; 24(Supl I): 5563.

21. Stein MB, Barret-Connor E. Quality of life in older adults receiving medications for anxiety, depression, or insomnia: findings from a community-based study. Am J Geriatr Psychiatry 2002; 10(5): 568-74.

22. Camarano, AA. Muito além dos 60: os novos idosos brasileiros. Rio de Janeiro: IPEA; 1999.

23. Ersek M, Turner JA, Cain KC, Kemp CA. Chronic pain selfmanagement for older adults: a randomized controlled. BMC Geriatr 2004; 4(1): 7.
24. Gazalle FK, Lima MS, Tavares BF, Hallal PC. Sintomas depressivos e fatores associados em população idosa no Sul do Brasil. Rev Saude Publica 2004; 38(3): 365-71.

25. Chin A Paw MJ, van Poppel MN, Twisc JW, van Mechelen W. Effects of resistance and all-round, functional training on quality of life, vitality and depression of older adults living in long-term care facilities: a 'randomized' controlled trial. BMC Geriatr 2004; 4(1): 5.

26. Strawbridge WJ, Deleger S, Roberts RE, Kaplan GA. Physical activity reduces the risk of subsequent depression for older adults. Am J Epidemiol 2002; 156(4): 328-34.

27. Van Gool CH, Kempen GI, Penninx BW, Deeg DJ, Beekman AT, van Eijk JT. Relationship between changes in depressive symptoms and unhealthy lifestyles in late middle aged and older persons: results from the Longitudinal Aging Study Amsterdam. Age and Ageing 2003; 32(1): 81-7.

28. Almeida OP. Idosos atendidos em serviço de emergência de saúde mental: características demográficas e clínicas. Rev Bras Psiquiatr 1999; 21(1): 12-18.

29. Szklo M, Nieto FJ. Epidemiology: beyond the basics. Boston: Jones and Barlett Publishers; 2004.

30. Reichenheim ME, Moraes CL. Alguns pilares para a apreciação da validade de estudos epidemiológicos. Rev Bras Epidemiol 1998; 1(2): 131-48.

recebido em: 28/11/05 versão final reapresentada em: 28/08/06 aprovado em: 29/09/06 